\title{
ESTIMACIÓN DE LA INCERTIDUMBRE EN LA ESTANDARIZACIÓN DE UNA SOLUCIÓN DE ÁCIDO SULFÚRICO
}

\author{
ESTIMATED UNCERTAINTY IN THE STANDARDIZATION OF SULFURIC ACID SOLUTION
}

\section{Gloría Inés GIRALDO GÓMEZ1, Diógenes de Jesús RAMíREZ RAMÍREZ1, Nancy Rocío SANABRIA GONZÁLEZ ${ }^{2 *}$}

${ }^{1}$ Facultad de Ciencias Exactas y Naturales, Universidad Nacional de Colombia Sede Manizales, Campus La Nubia, Km 4 Vía al Magdalena, AA 127 Manizales, Colombia.

2 Facultad de Ingeniería y Arquitectura, Universidad Nacional de Colombia Sede Manizales, Campus la Nubia, Km 4 Vía al Magdalena, AA 127 Manizales, Colombia.
Historia del Artículo

Recibido: Agosto 09, 2016

Evaluado: Diciembre 20, 2016

Aceptado: Enero 12, 2017

Disponible: Febrero 07, 2017

*Autor Corresponsal. E-mail: nrsanabriag@unal.edu.co

Resumen |

Se estimó la incertidumbre de medición en la concentración de una solución de ácido sulfúrico $\left(\mathrm{H}_{2} \mathrm{SO}_{4}\right)$ estandarizada con un patrón primario de carbonato de sodio anhidro $\left(\mathrm{Na}_{2} \mathrm{CO}_{3}\right)$, mediante un ejemplo sencillo de procesamiento de los datos experimentales obtenidos en el laboratorio. La metodología empleada se basó en la guía establecida por la EURACHEM/CITAC e incluyó las siguientes etapas: identificación de las fuentes de incertidumbre, evaluación de la incertidumbre estándar y cálculo de la incertidumbre estándar combinada e incertidumbre expandida. Las fuentes que presentaron mayor contribución a la incertidumbre fueron la reproducibilidad y la repetibilidad provenientes de la medición del volumen de $\mathrm{H}_{2} \mathrm{SO}_{4}$ gastado en la valoración del $\mathrm{Na}_{2} \mathrm{CO}_{3}$. Bajo las condiciones de estudio, la incertidumbre en la estandarización de una solución de $\mathrm{H}_{2} \mathrm{SO}_{4}$ de concentración 0.0127 $\mathrm{mol} / \mathrm{L}$ fue $\pm 0.0015 \mathrm{~mol} / \mathrm{L}$.

Palabras Clave: Incertidumbre de la medición; estandarización; Ácido sulfúrico; titulación.

\section{Abstract}

The uncertainty of measurement in the concentration of a solution of sulfuric acid $\left(\mathrm{H}_{2} \mathrm{SO}_{4}\right)$ standardized with a primary standard of anhydrous sodium carbonate $\left(\mathrm{Na}_{2} \mathrm{CO}_{3}\right)$ was estimated by using a simple example of processing of the experimental data obtained in the laboratory. The methodology used was based on the guidelines of the EURACHEM/CITAC, including the following steps: identification of sources of uncertainty, assessment of standard uncertainty and calculation of combined standard uncertainty and expanded uncertainty. The sources that mostly contributed to uncertainty were the reproducibility and repeatability arising from measuring the volume of $\mathrm{H}_{2} \mathrm{SO}_{4}$ used in the standardization of $\mathrm{Na}_{2} \mathrm{CO}_{3}$. Under the study conditions, the uncertainty in the standardization of a solution of $\mathrm{H}_{2} \mathrm{SO}_{4}$ in a concentration of $0.0127 \mathrm{~mol} / \mathrm{L}$ was $\pm 0.0015 \mathrm{~mol} / \mathrm{L}$.

Keywords: Measurement uncertainty; standardization; sulfuric acid; titration.

\section{INTRODUCCIÓN |}

Una de las actividades habituales en los laboratorios de química es la preparación de soluciones ácidas o básicas de concentración conocida, mediante la dilución de una cantidad determinada del reactivo analítico con agua destilada en un balón volumétrico aforado. Los reactivos analíticos comerciales usados regularmente en la preparación de estas soluciones son $\mathrm{NaOH}, \mathrm{KOH}, \mathrm{HCl}$ y $\mathrm{H}_{2} \mathrm{SO}_{4}$, los cuales no son patrones primarios y por tanto, la concentración de las soluciones preparadas es aproximada, siendo necesario realizar una estandarización. Adicional a la estandarización de la solución, es importante conocer el grado de confianza del resultado de la concentración, es decir, establecer cuanto puede alejarse el resultado analítico del valor considerado verdadero mediante la estimación de la incertidumbre de medida.

El término incertidumbre de medida se ha definido como un "parámetro asociado al resultado de una medida, que caracteriza la dispersión de los valores que podrían razonablemente ser atribuidos al mensurando". "La incertidumbre de medida no implica duda sobre la validez de una medida; al contrario, el conocimiento de la incertidumbre 
implica un aumento de la confianza en la validez del resultado de una medición" (EURACHEM/CITAT, 2012).

La solución estandarizada de ácido sulfúrico $\left(\mathrm{H}_{2} \mathrm{SO}_{4}\right)$ tiene numerosas aplicaciones en los laboratorios de análisis químico, por ejemplo, en la cuantificación de alcalinidad del agua, determinación de nitrógeno Kjeldahl en proteínas y en general en las valoraciones ácido-base. Por tanto, es necesario evaluar la incertidumbre asociada a la estandarización de esta solución y así garantizar confiabilidad en los resultados analíticos obtenidos.

Aunque existen metodologías para la estimación de la incertidumbre en mediciones analíticas (Evans et al, 1984; Rozet et al, 2011; EURACHEM/CITAT, 2012), la evaluación de la incertidumbre asociada a una medición es compleja, debido al elevado número de fuentes de error en los procedimientos analíticos (Maroto Sánchez, 2002) y a que se requiere información de diferentes tipos, como datos técnicos de los equipos, certificados de calibración, ensayos de repetibilidad del método, entre otros; esto sin mencionar el conocimiento de interpretación de los mismos (Delgado Alamilla et al, 2005). En la literatura científica se encuentran ejemplos de estimación de la incertidumbre en las mediciones de plomo en sangre por voltamperometría de redisolución anódica (Delgado et al, 2013), proteínas en alimentos por el método de Kjeldahl (Vano et al, 2011), hidrocarburos aromáticos policíclicos en lodos de aguas residuales industriales (Pereyra et al, 2015), cobre por espectroscopía de absorción atómica (Morales Ramírez et al, 2012), reflexión de fluorescencia de rayos $X$ (Floor et al, 2015) y antioxidantes fenólicos en aceites (Kim et al, 2016), entre otros. Sin embargo, un gran número de los artículos no muestra en detalle los procedimientos realizados y los datos completos a partir de los cuales se realizaron los cálculos para la estimación de la incertidumbre.

En este trabajo se estimó la incertidumbre de medición en la estandarización de una solución de $\mathrm{H}_{2} \mathrm{SO}_{4}$, mediante un ejemplo sencillo de procesamiento de los datos experimentales obtenidos en el laboratorio, basado en la metodología establecida por la EUROCHEM/CITAC (2012). El trabajo promueve la articulación de la teoría con la práctica y el entorno real, y muestra con detalle los cálculos realizados en la cuantificación de la incertidumbre.

\section{MATERIALES Y MÉTODOS}

\section{Reactivos}

$\mathrm{H}_{2} \mathrm{SO}_{4}$ (Merck, 98\%, $\rho=1.84 \mathrm{~g} / \mathrm{cm}^{3}$ ), $\mathrm{Na}_{2} \mathrm{CO}_{3}$ anhidro (Merck, 99.9\%, tolerancia en la pureza de $\pm 0.5 \%$ ), agua destilada y desionizada (conductividad $<3 \mu \mathrm{mhos} / \mathrm{cm}$ ).

\section{Equipos}

Balanza analítica digital (Mettler Toledo, modelo $A B$ 204), rango de medición de 0.01 a $210 \mathrm{~g}$ y resolución de $0.0001 \mathrm{~g}$. Los resultados de la calibración de la balanza para una carga de $0.01 \mathrm{~g}$ fueron: desviación de $0.00007 \mathrm{~g}$ e incertidumbre de $0.0002 \mathrm{~g}$. Para una carga de $100 \mathrm{~g}$ la desviación e incertidumbre fueron de 0.00005 y $0.0003 \mathrm{~g}$, respectivamente.

Titulador automático Metrohm (Mettler Toledo, modelo SM Titrino 702), resolución de $0.0001 \mathrm{~mL}$. Los resultados del test de volumetría para buretas Metrohm fueron los siguientes: error absoluto de $24.35 \mu \mathrm{L}$, límite error absoluto de $40.00 \mu \mathrm{L}$ y límite error relativo $0.2 \%$.

\section{Procedimiento para la estandarización de la solución de} $\mathrm{H}_{2} \mathrm{SO}_{4}$

Se utilizaron soluciones de ácido sulfúrico $\left(\mathrm{H}_{2} \mathrm{SO}_{4}\right)$ con una concentración aproximada de $0.0125 \mathrm{~mol} / \mathrm{L}$. La solución de $\mathrm{H}_{2} \mathrm{SO}_{4}$ se estandarizó frente al patrón primario de carbonato de sodio anhidro $\left(\mathrm{Na}_{2} \mathrm{CO}_{3}\right)$. El $\mathrm{Na}_{2} \mathrm{CO}_{3}$ se secó en la estufa entre 180 y $200{ }^{\circ} \mathrm{C}$, se llevó a un desecador, luego se pesó $0.0100 \pm 0.0001 \mathrm{~g}$ y se disolvieron en $100 \mathrm{~mL}$ de agua. Esta solución se valoró en el titulador automático hasta neutralización usando la solución de $\mathrm{H}_{2} \mathrm{SO}_{4}$. Para la titulación del blanco se tomaron $100 \mathrm{~mL}$ de agua destilada y se valoraron en el mismo titulador automático usando la solución de $\mathrm{H}_{2} \mathrm{SO}_{4}$.

\section{Evaluación de la reproducibilidad y la repetibilidad}

Con el fin de analizar la reproducibilidad de las medidas, el procedimiento para la estandarización de la solución de $\mathrm{H}_{2} \mathrm{SO}_{4}$ se repitió 30 veces frente al patrón primario $\mathrm{Na}_{2} \mathrm{CO}_{3}$ (3 ensayos por mes) y 15 veces para el blanco (1 ensayo cada 20 días). Adicionalmente, en un día, el mismo operario y bajo las mismas condiciones de medida, realizó 5 ensayos para determinar la repetibilidad de los procedimientos.

\section{Estimación de la incertidumbre}

Las etapas seguidas para la estimación de la incertidumbre en la estandarización de una solución de $\mathrm{H}_{2} \mathrm{SO}_{4}$, acordes con la metodología establecida por la EUROCHEM/CITAC (2012) se resumen a continuación:

- Especificación el mensurando.

- Identificación las fuentes de incertidumbre relevantes.

- Cuantificación de los componentes de la incertidumbre.

- Calculo de la incertidumbre combinada e incertidumbre expandida.

En este trabajo no se consideraron las fuentes de incertidumbre concernientes a la preparación de la solución de $\mathrm{H}_{2} \mathrm{SO}_{4}$, la sensibilidad de la balanza y las variaciones por temperatura. Como la concentración de $\mathrm{H}_{2} \mathrm{SO}_{4}$ se determina mediante valoración con un patrón primario $\left(\mathrm{Na}_{2} \mathrm{CO}_{3}\right)$ y no por cálculo directo, no se requiere información sobre las fuentes de incertidumbre relacionadas con el volumen de $\mathrm{H}_{2} \mathrm{SO}_{4}$ medido, la pureza del reactivo y la masa molar del $\mathrm{H}_{2} \mathrm{SO}_{4}$ (EURACHEM/CITAT, 2012). La medición de la masa involucra como fuente de incertidumbre la función de calibración y esta a su vez depende de la sensibilidad de la balanza y su linealidad. La sensibilidad puede despreciarse 
porque la masa se calcula por diferencia de dos pesadas en la misma balanza en un rango muy estrecho (EURACHEM/CITAT, 2012). Las variaciones en la masa debidas a cambios en la temperatura no se analizaron, por cuanto la balanza empleada dispone de un sistema de autoajuste para compensar la deriva térmica residual (Mettler Toledo, 2001). Respecto al titulador, este realiza compensación automática de la temperatura en las medidas de pH (Metrohm, 2012).

\section{RESULTADOS Y DISCUSIÓN |}

\section{Identificación del mensurando}

Se refiere a la magnitud a la cual se le quiere estimar la incertidumbre, en este caso el mensurado corresponde a la concentración de la solución de $\mathrm{H}_{2} \mathrm{SO}_{4}$ utilizada. Generalmente el mensurando $(y)$ no se mide directamente, sino que se determina a partir de otras magnitudes mediante una función modelo.

Función modelo para el cálculo de la molaridad de la solución de $\mathrm{H}_{2} \mathrm{SO}_{4}$

La ecuación utilizada para determinar la concentración de la solución de $\mathrm{H}_{2} \mathrm{SO}_{4}$ es la siguiente:

$C=\frac{m_{\mathrm{Na}_{2} \mathrm{Co}_{3} \times \mathrm{Pa}_{2} \mathrm{Co}_{3} \times 1000}}{\mathrm{M}_{\mathrm{Na}} \mathrm{Co}_{3} \times\left(\mathrm{V}_{1}-\mathrm{V}_{0}\right)}$

donde:

$\mathrm{C}=$ concentración molar de la solución de $\mathrm{H}_{2} \mathrm{SO}_{4}$, $\mathrm{mol} / \mathrm{L}$.

$m_{\mathrm{Na}_{2} \mathrm{CO}_{3}}=$ masa de $\mathrm{Na}_{2} \mathrm{CO}_{3}$, g.

$P_{\mathrm{Na}_{2} \mathrm{CO}_{3}}=$ Pureza del reactivo $\mathrm{Na}_{2} \mathrm{CO}_{3}$, fracción en masa.

$\mathrm{M}_{\mathrm{Na}_{2} \mathrm{CO}_{3}}=$ Masa molar del $\mathrm{Na}_{2} \mathrm{CO}_{3}, 105.9884 \mathrm{~g} / \mathrm{mol}$.

$V_{1}=$ Volumen de $\mathrm{H}_{2} \mathrm{SO}_{4}$ empleado en la titulación del patrón de $\mathrm{Na}_{2} \mathrm{CO}_{3}$, $\mathrm{mL}$

$V_{0}=$ Volumen de $\mathrm{H}_{2} \mathrm{SO}_{4}$ empleado en la titulación del agua destilada (blanco), $\mathrm{mL}$

$1000=$ es un factor de conversión de unidades de $\mathrm{mL}$ a L.

\section{Identificación de las magnitudes de entrada}

De acuerdo con el modelo matemático para el cálculo de la molaridad de la solución de $\mathrm{H}_{2} \mathrm{SO}_{4}$, las magnitudes de entrada corresponden a la masa de $\mathrm{Na}_{2} \mathrm{CO}_{3}$, el volumen de la solución de $\mathrm{H}_{2} \mathrm{SO}_{4}$ gastado en la titulación del patrón de $\mathrm{Na}_{2} \mathrm{CO}_{3}$ y el volumen de la solución de $\mathrm{H}_{2} \mathrm{SO}_{4}$ gastado en la titulación del blanco. Las constantes de entrada son el factor de conversión de $\mathrm{mL}$ a $\mathrm{L}$ (constante de 1000) y el peso molecular del $\mathrm{Na}_{2} \mathrm{CO}_{3}$. En la Tabla 1 y Tabla 2 se muestran los resultados de las valoraciones realizadas al $\mathrm{Na}_{2} \mathrm{CO}_{3}$ y al blanco (ensayos de reproducibilidad). En la Tabla 3 se observan los resultados de las repeticiones de las valoraciones, tanto al patrón de $\mathrm{Na}_{2} \mathrm{CO}_{3}$ como al blanco.

\section{Fuentes de incertidumbre}

En el diagrama causa-efecto (Fig. 1) se muestran las diferentes fuentes de incertidumbre asociadas a cada magnitud de entrada.

\begin{tabular}{|c|c|c|c|c|c|}
\hline $\begin{array}{c}m_{\mathrm{Na}_{2} \mathrm{CO}_{3}} \\
\text { (g) }\end{array}$ & $\begin{array}{c}V_{1} \\
(\mathrm{~mL})\end{array}$ & $\begin{array}{c}m_{\mathrm{Na}_{2} \mathrm{CO}_{3}} \\
(\mathrm{~g})\end{array}$ & $\begin{array}{c}V_{1} \\
(\mathrm{~mL})\end{array}$ & $\begin{array}{c}m_{\mathrm{Na}_{2} \mathrm{CO}_{3}} \\
(\mathrm{~g})\end{array}$ & $\begin{array}{c}V_{1} \\
(\mathrm{~mL})\end{array}$ \\
\hline 0.0099 & 7.898 & 0.0100 & 8.256 & 0.0101 & 7.617 \\
\hline 0.0101 & 7.773 & 0.0101 & 7.918 & 0.0100 & 7.679 \\
\hline 0.0100 & 7.578 & 0.0100 & 7.329 & 0.0100 & 7.800 \\
\hline 0.0099 & 7.499 & 0.0101 & 7.159 & 0.0101 & 7.383 \\
\hline 0.0099 & 7.868 & 0.0099 & 6.967 & 0.0099 & 7.116 \\
\hline 0.0100 & 7.734 & 0.0100 & 7.188 & 0.0099 & 7.669 \\
\hline 0.0101 & 7.669 & 0.0099 & 7.479 & 0.0100 & 7.805 \\
\hline 0.0101 & 7.695 & 0.0099 & 7.885 & 0.0101 & 7.726 \\
\hline 0.0101 & 7.891 & 0.0097 & 7.710 & 0.0104 & 7.886 \\
\hline 0.0101 & 8.013 & 0.0099 & 7.462 & 0.0105 & 8.050 \\
\hline \multicolumn{6}{|c|}{$V_{0}(\mathrm{~mL})$} \\
\hline 0.140 & 0.14 & & & 0.134 & 0.148 \\
\hline 0.144 & 0.15 & & & 0.136 & 0.140 \\
\hline 0.137 & 0.13 & & & 0.140 & 0.161 \\
\hline
\end{tabular}

Tabla 3. Masa de $\mathrm{Na}_{2} \mathrm{CO}_{3}$ y volumen de solución de $\mathrm{H}_{2} \mathrm{SO}_{4}$ empleado en las valoraciones - Ensayos de repetibilidad

\begin{tabular}{ccc}
\hline \multicolumn{2}{c}{ Titulación del patrón } & Blanco \\
\hline$m_{\mathrm{Na}_{2} \mathrm{CO}_{3}}$ & $V_{1}$ & $V_{0}$ \\
$(\mathrm{~g})$ & $(\mathrm{mL})$ & $(\mathrm{mL})$ \\
0.0101 & 7.824 & 0.140 \\
0.0100 & 7.266 & 0.144 \\
0.0100 & 7.572 & 0.153 \\
0.0099 & 7.414 & 0.141 \\
0.0100 & 7.922 & 0.139 \\
\hline
\end{tabular}

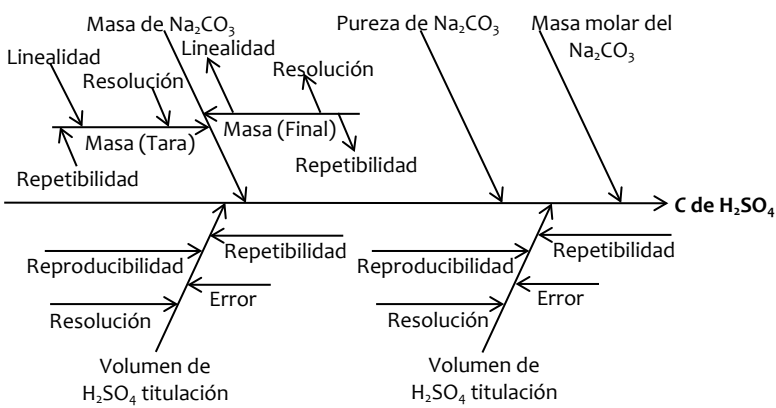

Figura 1. Diagrama causa - efecto para la estandarización de la solución de $\mathrm{H}_{2} \mathrm{SO}_{4}$

Los componentes de la incertidumbre se pueden agrupar en dos categorías según el método de evaluación, tipo A y tipo B. La evaluación tipo A se utiliza cuando se han realizado $n$ observaciones independientes de una de las magnitudes de entrada, bajo las mismas condiciones de medida. La evaluación tipo B se emplea cuando la estimación de una magnitud de entrada no ha sido obtenida a partir de observaciones repetidas, este conjunto de información puede incluir: certificados de calibración, especificaciones del instrumento, valores de mediciones anteriores $y$ conocimiento sobre las características o el comportamiento del sistema de medición (EURACHEM/CITAT, 2012). 
La cuantificación de una fuente de incertidumbre incluye la asignación de un valor y la determinación de la distribución a la cual se refiere este valor. Las distribuciones más utilizadas son: normal, rectangular, triangular y t-student. En la Tabla 4 se resumen los tipos de incertidumbre y las distribuciones de probabilidad para la determinación de la concentración del $\mathrm{H}_{2} \mathrm{SO}_{4}$.

\begin{tabular}{|c|c|c|c|c|c|}
\hline \multicolumn{2}{|c|}{$\begin{array}{c}\text { Magnitud de } \\
\text { entrada }\end{array}$} & $\begin{array}{c}\text { Fuente de } \\
\text { incertidumbre }\end{array}$ & $\begin{array}{c}\text { Tipo de } \\
\text { incertidumbre }\end{array}$ & Distribución & Unidad \\
\hline \multirow{6}{*}{$\begin{array}{l}\text { Masa de } \\
\mathrm{Na}_{2} \mathrm{CO}_{3}\end{array}$} & \multirow{3}{*}{ Tara } & Repetibilidad & Tipo A & Normal & $\bar{g}$ \\
\hline & & Resolución & Tiрo B & Rectangular & $\mathrm{g}$ \\
\hline & & Linealidad & Tipo B & Rectangular & $\mathrm{g}$ \\
\hline & \multirow{3}{*}{ Final } & Repetibilidad & Tipo A & Normal & $\mathrm{g}$ \\
\hline & & Resolución & Tipo B & Rectangular & $\mathrm{g}$ \\
\hline & & Linealidad & Tipo B & Rectangular & $\mathrm{g}$ \\
\hline \multirow{2}{*}{\multicolumn{2}{|c|}{ Pureza del $\mathrm{Na}_{2} \mathrm{CO}_{3}$}} & & Tipo B & Rectangular & $\%$ \\
\hline & & Repetibilidad & Tipo A & Normal & $\mathrm{mL}$ \\
\hline \multirow{7}{*}{$\begin{array}{l}\text { Volumen } \\
\text { de } \mathrm{H}_{2} \mathrm{SO}_{4}\end{array}$} & \multirow{2}{*}{ Patrón } & Reproducibilidad & Tipo A & Normal & $\mathrm{mL}$ \\
\hline & & Resolución & Tіро B & Rectangular & $\mathrm{mL}$ \\
\hline & \multirow{5}{*}{ Blanco } & Error & Tipo B & Rectangular & $\mathrm{mL}$ \\
\hline & & Repetibilidad & Tipo A & Normal & $\mathrm{mL}$ \\
\hline & & Reproducibilidad & Tipo A & Normal & $\mathrm{mL}$ \\
\hline & & Resolución & Tiрo B & Rectangular & $\mathrm{mL}$ \\
\hline & & Error & Tipo B & Rectangular & $\mathrm{mL}$ \\
\hline
\end{tabular}

Cálculo de la incertidumbre estándar e incertidumbre estándar combinada, $\boldsymbol{u}_{\boldsymbol{c}}(\boldsymbol{y})$

La incertidumbre estándar, $u(x)$, es la incertidumbre del resultado de una medición expresado como una desviación estándar. Una vez estimados los componentes individuales de la incertidumbre, el siguiente paso es calcular la incertidumbre estándar combinada de un valor y usando la siguiente expresión:

$$
u_{c}\left(y\left(x_{1}, x_{2}, \ldots\right)\right)=\sqrt{\sum_{i=1}^{n} c_{i}^{2} \times u\left(x_{i}\right)^{2}}
$$

donde $y\left(x_{1}, x_{2}, \ldots\right)$ es una función de varios parámetros $x_{1}, x_{2}, \ldots, c_{i}$ es un coeficiente de sensibilidad evaluado como $c_{i}=\frac{\partial y}{\partial x_{i}}$, y $u\left(y, x_{i}\right)$ denota la incertidumbre en $x_{i}$. La expresión para combinar incertidumbres se puede reducir a formas más sencillas, aplicando las siguientes reglas (EURACHEM/CITAT, 2012):

- Para modelos que involucren sumas o diferencias de cantidades, por ejemplo $y=(p+q+r+\cdots)$, la incertidumbre estándar combinada $u_{c}(y)$ viene dada por:

$\boldsymbol{u}_{c}(\boldsymbol{y}(\boldsymbol{p}, \boldsymbol{q} \ldots))=\sqrt{\boldsymbol{u}(\boldsymbol{p})^{2}+\boldsymbol{u}(\boldsymbol{q})^{2}+\cdots}$

- Para modelos que involucren productos y cocientes, por ejemplo $y=(p \times q \times r \times \ldots)$ o $y=p /(q \times r \times \ldots)$, la incertidumbre estándar combinada $u_{c}(y)$ viene dada por:

$u_{c}(y)=y \sqrt{\left(\frac{u(p)}{p}\right)^{2}+\left(\frac{u(q)}{q}\right)^{2}+\cdots}$
A continuación se especifica cómo se realizó el cálculo de la incertidumbre estándar e incertidumbre estándar combinada para cada fuente de las magnitudes de entrada.

Masa de $\mathrm{Na}_{2} \mathrm{CO}_{3}$ : esta magnitud tiene 3 fuentes de incertidumbre debidas a la resolución, linealidad y repetibilidad. La resolución de la balanza es $0.0001 \mathrm{~g}$. Del certificado de calibración de la balanza se obtienen los datos incertidumbre (0.0002 g) y de repetibilidad (0.00007 g) para una carga de $0.01 \mathrm{~g}$. En la Tabla 5 se resumen los resultados obtenidos.

Tabla 5. Valores e incertidumbre en la masa de $\mathrm{Na}_{2} \mathrm{CO}_{3}$

\begin{tabular}{ccccc} 
Fuente & $\begin{array}{c}\text { Valor, } \\
\boldsymbol{x}\end{array}$ & $\begin{array}{c}\text { Incertidumbre, } \\
\boldsymbol{u}\end{array}$ & $\begin{array}{c}\text { Incertidumbre } \\
\text { estándar, } \boldsymbol{u}(\boldsymbol{x})\end{array}$ & $\boldsymbol{u}(\boldsymbol{x})^{2}$ \\
\hline Resolución & 0.0001 & & $\frac{0.0001}{\sqrt{3}}$ & $3.3333 \times 10^{-9}$ \\
Linealidad & & 0.0002 & $\frac{0.0002}{2}$ & $1.0000 \times 10^{-8}$ \\
Repetibilidad & & & 0.00007 & $\begin{array}{c}4.9000 \times 10^{-9} \\
\end{array}$ \\
& & & $\sum u_{x}^{2}=1.8233 \times 10^{-8}$ \\
\hline
\end{tabular}

La masa de $\mathrm{Na}_{2} \mathrm{CO}_{3}$ tiene dos contribuciones, la masa de la tara y la masa final. La incertidumbre estándar combinada $u_{c}(m)$ se obtiene por la aplicación de la ecuación (3):

$u_{c}(\boldsymbol{m})=\boldsymbol{u}_{c}\left(\boldsymbol{m}_{\mathrm{Na}_{2} \mathrm{CO}_{3}}\right)=\sqrt{\boldsymbol{u}\left(\boldsymbol{m}_{\text {tara }}\right)^{2}+\boldsymbol{u}\left(\boldsymbol{m}_{\text {final }}\right)^{2}}$

Como las dos mediciones fueron realizadas en la misma balanza:

$u_{c}(m)=\sqrt{2 \times u\left(m_{\text {final }}\right)^{2}}=\sqrt{2 \times 1.8233 \times 10^{-8}}=1.9096 \times 10^{-4}$. (6)

Pureza del $\mathrm{Na}_{2} \mathrm{CO}_{3}$ : de los datos de la etiqueta del reactivo se obtiene una tolerancia de $\pm 0.5 \%$. Se asume una distribución rectangular y el valor de la incertidumbre $u(P)$ se calcula como $\frac{0.005}{\sqrt{3}}=2.8868 \times 10^{-3}$.

Masa molecular del $\mathrm{Na}_{2} \mathrm{CO}_{3}$ : las masas atómicas poseen una incertidumbre reportada por la IUPAC (Wieser y Berglund, 2009). Para calcular la incertidumbre estándar se asume una distribución rectangular, es decir se divide el valor entre $\sqrt{3}$. En la Tabla 6 se muestran los resultados obtenidos.

Tabla 6. Valores e incertidumbre en la masa molecular del $\mathrm{Na}_{2} \mathrm{CO}_{3}$

\begin{tabular}{|c|c|c|c|c|c|}
\hline Elemento & $\mathrm{N}^{\circ}$ & Incertidumbre & $\begin{array}{l}\text { Incertidumbre } \\
\text { estándar, } u(x) \\
\end{array}$ & $u(x)^{2}$ & $u(x)^{2}$ total \\
\hline $\mathrm{Na}$ & 2 & 0.00000002 & $1.1547 \times 10^{-8}$ & $1.3333 \times 10^{-16}$ & $2.6667 \times 10^{-16}$ \\
\hline$C$ & 1 & 0.0008 & $4.6188 \times 10^{-4}$ & $2.1333 \times 10^{-7}$ & $2.1333 \times 10^{-7}$ \\
\hline 0 & 3 & 0.0003 & $1.7321 \times 10^{-4}$ & $\begin{array}{r}3.0000 \times 10^{-8} \\
\sum u_{x}^{2}=\end{array}$ & $\begin{array}{l}9.0000 \times 10^{-8} \\
333 \times 10^{-7}\end{array}$ \\
\hline
\end{tabular}

La incertidumbre estándar combinada $u(M)$ viene dada por:

$$
\begin{aligned}
& u_{c}(M)=u_{c}\left(M_{N_{2} C_{2} O_{3}}\right)=\sqrt{2 \times u\left(M_{N a}\right)^{2}+1 \times u\left(M_{C}\right)^{2}+3 \times u\left(M_{o}\right)^{2}} \\
& =5.5075 \times 10^{-4}
\end{aligned}
$$

Volumen de $\mathrm{H}_{2} \mathrm{SO}_{4}$ empleado en la estandarización del patrón: las fuentes de incertidumbre asociadas a esta magnitud son resolución, reproducibilidad, repetibilidad y error absoluto (del volumen nominal de cilindro proveniente del certificado de calibración). Las desviaciones estándar de 
las 30 mediciones (ver Tabla 1) y de las 5 mediciones (ver Tabla 3) fueron 0.2973 y $0.2742 \mathrm{~mL}$, respectivamente. En la Tabla 7 se resumen los valores obtenidos.

Volumen de $\mathrm{H}_{2} \mathrm{SO}_{4}$ empleado en la titulación del blanco: las fuentes de incertidumbre asociadas a esta magnitud son las mismas que para el volumen de $\mathrm{H}_{2} \mathrm{SO}_{4}$ empleado en la estandarización del patrón. Las desviaciones estándar de las 15 mediciones (ver Tabla 2) y de las 5 repeticiones (ver Tabla 3) fueron de $7.6457 \times 10^{-3}$ y $5.6833 \times 10^{-3} \mathrm{~mL}$, respectivamente. En la Tabla 8 se muestran los resultados obtenidos.

Tabla 7. Valores e incertidumbre en el volumen de $\mathrm{H}_{2} \mathrm{SO}_{4}$ empleado en la estandarización

\begin{tabular}{cccc}
\hline Fuente & Valor, $\boldsymbol{x}$ & $\begin{array}{l}\text { Incertidumbre } \\
\text { estándar, } \boldsymbol{u}(\boldsymbol{x})\end{array}$ & $\boldsymbol{u}(\boldsymbol{x})^{\mathbf{2}}$ \\
\hline Resolución & 0.0001 & $\frac{0.0001}{\sqrt{3}}$ & $3.3333 \times 10^{-9}$ \\
Reproducibilidad & & 0.2973 & $8.8387 \times 10^{-2}$ \\
Repetibilidad & & 0.2742 & $7.5186 \times 10^{-2}$ \\
Error & 0.02435 & $\frac{0.02435}{\sqrt{3}}$ & $1.9764 \times 10^{-4}$ \\
& & & $\sum u_{x}^{2}=1.6377 \times 10^{-1}$ \\
\hline
\end{tabular}

Tabla 8. Valores e incertidumbre en el volumen de $\mathrm{H}_{2} \mathrm{SO}_{4}$ empleado la titulación del blanco

\begin{tabular}{cccc}
\hline \multicolumn{4}{c}{ titulación del blanco } \\
\hline Fuentes & Valor, $\boldsymbol{x}$ & $\begin{array}{l}\text { Incertidumbre } \\
\text { estándar, } \boldsymbol{u}(\boldsymbol{x})\end{array}$ & $\boldsymbol{u}(\boldsymbol{x})^{\mathbf{2}}$ \\
\hline Resolución & 0.0001 & $\frac{0.0001}{\sqrt{3}}$ & $3.3333 \times 10^{-9}$ \\
Reproducibilidad & & $7.6457 \times 10^{-3}$ & $5.8457 \times 10^{-5}$ \\
Repetibilidad & & $5.6833 \times 10^{-3}$ & $3.2300 \times 10^{-5}$ \\
Error & 0.02435 & $\frac{0.02435}{\sqrt{3}}$ & $1.9764 \times 10^{-4}$ \\
& & & $\sum u_{x}^{2}=2.8840 \times 10^{-4}$ \\
\hline
\end{tabular}

La incertidumbre estándar combinada asociada al volumen real en la estandarización de la solución $u_{c}(V)$ viene dada por:

$u_{c}(V)=\sqrt{u\left(V_{1}\right)^{2}+u\left(V_{0}\right)^{2}}$

Aplicando nuevamente la ecuación (3) se tiene que:

$u_{c}(V)=\sqrt{1.6377 \times 10^{-1}+2.8840 \times 10^{-4}}$

$=4.0504 \times 10^{-1}(9)$

Calculo de la incertidumbre combinada de la concentración: la incertidumbre estándar combinada $u_{c}(C)$ para la función modelo del cálculo de la concentración de la solución de $\mathrm{H}_{2} \mathrm{SO}_{4}$ viene dada por:

$$
u_{c}(C)=C \sqrt{\left(\frac{u_{c}(m)}{m}\right)^{2}+\left(\frac{u(P)}{P}\right)^{2}+\left(\frac{u_{c}(M)}{M}\right)^{2}+\left(\frac{u_{c}(V)}{V}\right)^{2}}
$$

Como un ejemplo de cálculo específico en la estandarización de una solución se emplearon $0.0100 \mathrm{~g}$ de $\mathrm{Na}_{2} \mathrm{CO}_{3}$ disueltos en $100 \mathrm{~mL}$ de agua y se gastaron $7.561 \mathrm{~mL}$ de solución de $\mathrm{H}_{2} \mathrm{SO}_{4}$. En la valoración del blanco se consumieron $0.148 \mathrm{~mL}$ de $\mathrm{H}_{2} \mathrm{SO}_{4}$. Para estos datos la concentración obtenida es la siguiente:

$$
C=\frac{0.0100 g \times 0.999 \times 1000 m L / L}{105.9884 g / m o l \times(7.561 m L-0.148 m L)}=0.0127 \mathrm{~mol} / \mathrm{L}
$$

y la incertidumbre estándar combinada $u_{c}(C)$ se calcula como:

$$
\begin{gathered}
u_{c}(c)=c \times \sqrt{\left(\frac{1.9096 \times 10^{-4}}{0.0100}\right)^{2}+\left(\frac{2.8868 \times 10^{-3}}{0.999}\right)^{2}+\left(\frac{5.5075 \times 10^{-4}}{105.9884}\right)^{2}+\left(\frac{4.0504 \times 10^{-1}}{7.413}\right)^{2}} \\
u_{c}(C)=0.0127 \times \sqrt{3.3584 \times 10^{-3}}=7.3599 \times 10^{-4}
\end{gathered}
$$

\section{Calculo de la incertidumbre expandida}

Es una cantidad que define un intervalo alrededor del resultado de una medición, y que se espera abarque una fracción grande de la distribución de valores que se podrían atribuir razonablemente al mensurando. La incertidumbre expandida se calcula mediante la ecuación:

$$
U(C)=u_{c}(C) \times t_{p}\left(v_{e f f}\right)
$$

donde $u_{c}(C)$ es la incertidumbre estándar combinada y $t_{p}\left(v_{\text {eff }}\right)$ es un factor derivado de la distribución $t$-student a un nivel de confianza $p$ y $v_{\text {eff }}$ grados de libertad. Por convención la incertidumbre expandida se determina para un nivel de confianza del $95 \%$, usando un factor de cobertura $\mathrm{k}=2$ (EURACHEM/CITAT, 2012).

$$
U(C)=7.3599 \times 10^{-4} \times 2=1.4720 \times 10^{-3}
$$

Por lo tanto, la incertidumbre en la estandarización de una solución de $\mathrm{H}_{2} \mathrm{SO}_{4}$ de concentración $0.0127 \mathrm{~mol} / \mathrm{L}$ fue \pm $0.0015 \mathrm{~mol} / \mathrm{L}$. En la Figura 2 se muestra un diagrama con las contribuciones a la incertidumbre en la estandarización de una solución de $\mathrm{H}_{2} \mathrm{SO}_{4}$ y se aprecia que la fuente con mayor contribución es el volumen de ácido empleado en la titulación. De las fuentes de incertidumbre asociadas a este volumen, la reproducibilidad y repetibilidad son las que contribuyen en mayor proporción (97.6\% para $\mathrm{V}_{1}$ y $48.6 \%$ para $\mathrm{V}_{\mathrm{o}}$ ), resultados acordes a lo reportado en la literatura (Wampfler y Rösslein, 2009).

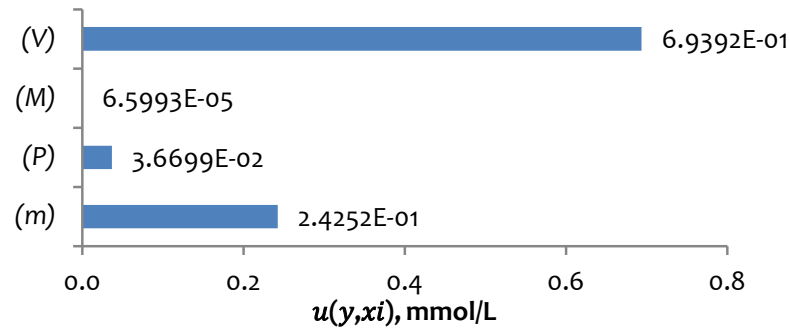

Figura 2. Contribuciones a la incertidumbre $u\left(y, x_{i}\right)$ en la estandarización de una solución de $\mathrm{H}_{2} \mathrm{SO}_{4}$

\section{CONCLUSIONES}

Para realizar una adecuada estimación de la incertidumbre de medida en la estandarización de una solución de $\mathrm{H}_{2} \mathrm{SO}_{4}$ es necesario conocer las fuentes de error en el método analítico empleado, aplicar conceptos básicos de estadística, disponer de los documentos de calibración de los equipos empleados y definir la metodología a seguir. La estimación de la incertidumbre en mediciones analíticas no sólo sirve para garantizar la calidad de la medición, sino que 
permite identificar las fuentes de error asociadas a esa medición y cuantificar su contribución.

La incertidumbre expandida relativa $(U(C) / C)$ en la estandarización de una solución de $\mathrm{H}_{2} \mathrm{SO}_{4}$, para un nivel de confianza del $95 \%$ con $k=2$ fue del $11.6 \%$. La fuente con mayor contribución a la incertidumbre fue el volumen de ácido empleado en la estandarización, siendo la reproducibilidad y repetibilidad las que aportan en mayor magnitud.

Mediante un ejemplo de aplicación sencillo, se logró articular la teoría sobre la metodología para la estimación de la incertidumbre en medidas analíticas con su aplicación en la estandarización de una solución de $\mathrm{H}_{2} \mathrm{SO}_{4}$.

\section{AGRADECIMIENTOS}

Los autores agradecen a la Universidad Nacional de Colombia Sede Manizales por el apoyo técnico para el desarrollo del presente trabajo.

\section{REFERENCIAS}

- Delgado Alamilla H., Itandehui Martínez Peña G.E., Pérez Salazar A. y Flores Flores M. 2005. Estimación de la incertidumbre en métodos de ensayos de construcción. Publicación Técnica No 275. Secretaria de Comunicaciones y Transportes, Instituto Mexicano del Transporte. Queretano - México, $88 \mathrm{p}$.

- Delgado G., Vanegas M. y Salazar J. 2013. Evaluación de la incertidumbre en la determinación de plomo en sangre por voltamperometría de redisolución anódica, aplicando el modelo de calibración ponderado de adición patrón generado por simulación de Monte Carlo. Universitas, 4: 58-70.

- EURACHEM/CITAT. 2012. Guide CG4 Quantifying Uncertainty in Analytical Measurement. S. L. R. Ellison y A. Williams (Eds). Third edition, 141 p.
- Evans J.S., Cooper D.W. y Kinney P. 1984. On the propagation of error in air pollution measurements. Environmental Monitoring and Assessment, 4: 139153.

- Floor G.H., Queralt I., Hidalgo M. y Marguí E. 2015. Measurement uncertainty in total reflection X-ray fluorescence. Spectrochimica Acta Part B: Atomic Spectroscopy, 111: 30-37.

- Kim J.-M., Choi S.-H., Shin G.-H., Lee J.-H., Kang S.-R., Lee K.-Y., Lim H.-S., Kang T.S. y Lee O.-H. 2016. Method validation and measurement uncertainty for the simultaneous determination of synthetic phenolic antioxidants in edible oils commonly consumed in Korea. Food Chemistry, 213: 19-25.

- Maroto Sánchez A. 2002. Incertidumbre en métodos analíticos de rutina. Tesis Doctoral. Departament de Química Analítica i Química Orgànica. Universitat Rovira I Virgili. Tarragona, España, $304 \mathrm{p}$

- Metrohm. 2012. Manual 702 SM Titrino. Applications. Herisau - Suiza, 90 p.

- Mettler Toledo. 2001. El ABC de la pesada. Greifensee -Switzerland, 12 p.

- Morales Ramírez A.J., Rángel Salinas E., Ramírez López A. y Dorantes Rosales H.J. 2012. Metodología para el cálculo de la incertidumbre en la determinación de cobre por espectroscopia de absorción atómica. Avances en Ciencias e Ingeniería, 3: 143-155.

- Pereyra M.T., Fernández Band B.S. y Lista A.G. 2015. Measurement uncertainty estimated from accuracy assessment by using an in-house reference material: two case studies for the extraction of total PAH in industrial wastewater sludges. Microchemical Journal, 119: 145-151.

- Rozet E., Rudaz S., Marini R.D., Ziémons E., Boulanger B. y Hubert P. 2011 Models to estimate overall analytical measurements uncertainty: Assumptions, comparisons and applications. Analytica Chimica Acta, 702: 160-171.

- Vano H., Kelim, Jiménez Y. y de Núñez M.G. 2011. Estimación de la incertidumbre de la medición para la determinación de proteínas en alimentos por el método de Kjeldahl. Revista Ingeniería UC, 18: 28-37.

- Wampfler B. y Rösslein M. 2009. Uncertainty due to volumetric operations is often underestimated. Talanta, 78: 113-119.

- Wieser M.E. y Berglund M. 2009. Atomic weights of the elements 2007 (IUPAC Technical Report). Pure and Applied Chemistry, 81: 2131-2156 